Accelerator Division

Alternating Gradient Synchrotron Department

BROOKHAVEN NATIONAL LABORATORY

Upton, New York 11973

Accelerator Division

Technical Note

AGS/AD/Tech. Note No. 359

\title{
SIEMENS UPGRADE CONTROL SYSTEM
}

Niels Schumburg

March 26, 1992

\section{INTRODUCTION}

The Siemens Motor Generator Power Supply System is being upgraded and modernized. Part of this process includes implementing a new control system, which is being modeled after the Booster Main Ring Power Supply Control System.

Because of the size and complexity of the project, it is being implemented in two stages. The first stage includes interfacing to the four power supply modules and the controlling PLC (programmable logic controller) for status and command interactions. The second stage will add controls for the exciter and the cycle converter.

It is the purpose of this draft to put down on paper all that has been agreed to through informal conversation and to solicit comments on this proposal.

\section{PROPOSED HARDWARE CONFIGURATION}

The Phase 1 system will have a single device controller that will communicate with an Apollo station via IEEE-488 and five remote VME interfaces via datacon. This system will be connected to the time line generator for AGS supercyle events and thus it will support multiple users (PPM). 
Four of the VME interfaces will be housed in four Siemens power supply racks. There will be four bipolar voltage functions and one unipolar current function required to control four Siemens power supply modules. The vector function generators will be used for this implementation. It uses a 12-bit DAC with an optional connection for an external 16-bit DAC.

For each outgoing function there will be the capability of digitizing and storing four incoming analog functions. The transient recorder will be used to obtain single timed readbacks or graphical snapshots of the analog functions. The sample rate is $10 \mathrm{kHz}$ and the storage capacity is $4 \mathrm{~K}$. The double function, VME interface, will have eight recorders and the rest, four.

The fifth VME interface will communicate with the master PLC and will support 48 statuses and the standard On, Off, and Standby command lines. The spill function will also be included in this bucket and will be one unipolar current function. It will have one vector function generator and four transient recorders. There will also be a scaler and two autodets used to globally stop all transient recorders simultaneously.

The Phase 2 system will add two remote VME interfaces to the phase 1 system. The exciter interface will have two vector function generators for one bipolar voltage and one unipolar current function. There will be eight transient recorders. The cycle converter interface will have three vector function generators for one unipolar speed, one unipolar real power and one unipolar reactive power functions. There will be ten transient recorders.

\section{PROPOSED FUNCTIONALITY}

\section{Single Timed Readback}

Each user will have the option of selecting a sample time, relative to AGS T0. For this time in the cycle, an averaged readback will be displayed. The average covers all cycles for each user. Since the sample clock is $10 \mathrm{kHz}$, there is a $100 \mu \mathrm{sec}$ time resolution. There will be 24 readbacks for Phase 1 and 42 total when Phase 2 is added.

\section{Vector Function Generators}

Each user will have the ability to download a vectorized version of any of the menu-selectable analog functions to the system. A vectorized copy of each function shall reside in what are called buffers in the device controller. They can be read and written to by an application program. Each user has a command that 
allows his function to be transferred to tables, which are on the vector function generators, in the remote interface. Tables can only be directly read, they cannot be directly loaded from an application program. Any one of four users, in synchronization with the timing system, can interface their analog functions to the Siemens system.

All of the Phase 1 system will have common vector timing. This includes four voltage, one current, and the spill functions. What this means is that the time duration for every vector for all six functions will be common. The Phase 2 system will have a separate common vector timing. This includes two exciter functions and three cycle converter functions.

Since each table is double buffered, new functions can be loaded asynchronously, while the old function is being generated. The transfer occurs in synchronization with the timing system so that the switch occurs at AGS T0 of the next cycle for that user.

There will be separate commands for the downloading of vectorized analog functions for the rectifiers (Phase 1) and the speed control (Phase 2) from the buffers to the tables. What this means, for example, is that while the power supply modules are generating their current and voltage outputs the speed control functions can be changed, downloaded, and generated independent of the rectifiers. The converse is also true, the rectifier functions can be modified independent of the speed control.

One of the features of the Booster Main Ring Power Supply was the ability to single cycle the system. A user could load into the buffers any group of functions and with the execution of this command, the power supply would generate one voltage and current copy. It would then rest at the last value of the functions, for all users, forever. A user could compel all other users to have a common dc or dwell value until commanded to do something else. This feature is also used to bring a system up gracefully to some dwell value prior to pulsing or down to the dwell prior to shut off. This feature will also be incorporated into this system. Since there are separate commands for speed and the rectifiers and the single cycle command execution effects both, it will not be a command option for both. The rectifier command options will be repetitive cycle and single cycle, and the speed command will only be repetitive cycle.

\section{$\underline{\text { PLC Interface }}$}

There will be a single master PLC that will interface to the control system. There will be 48 statuses monitored and the conventional On, Off, and Standby commands will be supported. 


\section{Transient Recorders}

Phase 1 will support 24 recorders associated with the rectifiers and Phase 2 will add up to 18 recorders for the speed control. With a sample rate of $10 \mathrm{kHz}$ and a storage capacity of $4 \mathrm{~K}$, when all recorders are simultaneously stopped, a 400 millisecond snapshot can be graphically displayed. Since this is inadequate, because of the duration of an AGS cycle, autodets are employed to stop digitization any variable time duration, into a cycle, from AGS T0. There are two different conditions under which stoppage occurs, programmatic and automatic because of a fault condition. The programmatic autodet is commanded On so a particular user can determine when all recorders stop. Other users are locked out. During the first cycle of the requesting user, a stop pulse is generated when the time duration has elapsed after AGS T0. It will globally stop all 42 recorders and they will be available for graphical display. The automatic autodet is commanded On so that if a fault occurs all recorders will stop. A scaler is used to determine where the fault occurred relative to AGS T0. The automatic autodet value determines how many post-fault samples are to occur before stoppage.

\section{OPERATIONAL PROCEDURE}

There is a need for an application program that will support the aforementioned functionality. A menu of desirable magnetic field functions should be available for operational selection. From this selection the program will vectorize the rectifier functions under the constraints imposed by the hardware and the designated parameters of the vectorizing algorithm. The hardware imposes a limit of 256 vectors, a maximum vector duration of 65.536 milliseconds, a variable slew rate per vector of 65.536 milliseconds, 16.384 milliseconds, or 4.096 milliseconds for full-scale variation ( $-10 \mathrm{VDC}$ to $+10 \mathrm{VDC})$, and a separate but common vector time duration for rectifier and speed functions.

There is a necessity, for Phase 2 operation, for the application program to also support speed functions that in conjunction with the rectifier functions are the underlying mechanism for controlling the Siemens motor generator. Some correlation between a selected $\mathrm{B}$ field, the derived five rectifier functions, and the exciter and cycle converter functions has to be determined. Since there does not have to be a fixed one-to-one relationship between the rectifier and speed functions, for a given rectifier function, the ability to support different speed functions is being proposed. The hardware has been proposed as having separate controls for both.

At this point in the project, the Phase 1 requirements seem to be straightforward and known. It is the integration of Phase 2 into the system where 
things look blurry. This includes the development of the speed algorithm, the relationship of the rectifier to the speed functions, and the implications of these unknowns on the functionality of the control system.

The programmatic and automatic (stop on fault) autodets should be armed and commanded from the application program. After stoppage of the transient recorders has occurred, any one should be selectable and graphically viewed.

\section{CONCLUSIONS}

A. The implementation of Phase 1 should be straightforward because it is a replica of a previously designed system.

B. The integration and implementation of Phase 2 is complex and, hence, requires additional thought and analysis.

C. The impact of Phase 2 on the functionality of the control system is not fully understood.

D. A person or group of individuals is needed to define the needs of the application program to fully run and operate the Siemens system.

mvh

schumb.tec 